\title{
Hemorragia suprarrenal en un recién nacido
}

\author{
Adrenal hemorrhage in a newborn
}

\author{
Luis Enrique Torres-Batista, ${ }^{*+}{ }^{+}$Fidel Enrique Torres-Batista, ${ }^{\ddagger}$ Idelvis Torres-Castañeda, ${ }^{\S}$ \\ Nancy de la Caridad-González,* Margarita Escalona-Fonseca* \\ * Departamento de Neonatología, Hospital Provincial Gineco-obstétrico “Fe del Valle Ramos”, Manzanillo; ${ }^{\ddagger}$ Hospital Provincial \\ “Carlos Manuel de Céspedes”, Bayamo; § Departamento de Enfermería, Policlínico No. 2 “Ángel Ortiz Vásquez”, Manzanillo, Cuba.
}

\begin{abstract}
RESUMEN
La hemorragia suprarrenal en el recién nacido se presenta en alrededor del $0.2 \%$; aproximadamente el $90 \%$ son unilaterales. Esta condición se asocia a traumatismo perinatal y a asfixia neonatal. Presentación del caso: Se trata de un recien nacido que nació por parto distócico por uso de fórceps, presentando depresión moderada (Apgar 1-7). A las 22 horas de vida se detecta dolor abdominal y mediante ultrasonido se diagnostica hemorragia suprarrenal del lado derecho. El manejo fue conservador y el paciente fue egresado sin complicaciones. Conclusiones: La hemorragia suprarrenal puede ser una entidad difícil para su detección; este caso puede ayudar a mejorar el proceso para su identificación y tratamiento.
\end{abstract}

Palabras clave: Hemorragia suprarrenal, recién nacido, masa abdominal, ultrasonografía.

\section{INTRODUCCIÓN}

La hemorragia suprarrenal (HS) es una entidad relativamente frecuente en el neonato, que por lo regular cursa de forma unilateral y asintomática, pudiendo incluso pasar desapercibida. La hemorragia en una o ambas glándulas suprarrenales tiene como principal factor patogénico el trauma obstétrico. Si bien la mayoría no suponen un compromiso

\footnotetext{
+ Correspondencia: LETB, luise@infomed.sld.cu

Conflicto de intereses: Los autores declaran que no tienen. Citar como: Torres-Batista LE, Torres-Batista FE, Torres-Castañeda I, De la Caridad-González N, Escalona-Fonseca M. Hemorragia suprarrenal en un recién nacido. Rev Mex Pediatr. 2020; 87(6): 218-220. https://dx.doi.org/10.35366/97683
}

\begin{abstract}
Adrenal hemorrhage in the newborn occurs in about $0.2 \%$; approximately $90 \%$ are unilateral. This condition is associated with perinatal trauma and neonatal asphyxia. Presentation of the case: Newborn who had a dystocic delivery due to the use of forceps, presenting moderate depression (Apgar 1-7). At 22 hours of life, abdominal pain was detected, and right-sided adrenal hemorrhage was diagnosed by ultrasound. Conservative management was provided and the patient was discharged without complications. Conclusions: The adrenal hemorrhage is a condition that can be difficult to detect; this report may help improve the process for its identification and treatment.
\end{abstract}

Keywords: Adrenal hemorrhage, newborn, abdominal mass, ultrasonography.

para el recién nacido $(\mathrm{RN})$, la posible dificultad en el diagnóstico y la ocasional evolución a insuficiencia suprarrenal aguda, hemorragia masiva o muerte hacen considerar a esta entidad de importancia significativa. ${ }^{1,2}$

La HS es la masa adrenal más frecuente en neonatos. Entre sus causas se incluyen: problemas durante el parto (por ejemplo, productos macrosómicos), asfixia, hipoxia, septicemia, trastornos de la coagulación, así como sífilis y tuberculosis..$^{3-5}$

En general, ocurre más frecuente en el lado derecho (70\%) y se atribuye a la compresión de la glándula suprarrenal entre el hígado y el riñón. La glándula suprarrenal es más susceptible a sufrir hemorragia durante la etapa neonatal como resultado de la regresión de la corteza fetal, que ocurre durante las primeras 
seis semanas de vida, debido a la ingurgitación de los vasos corticales. ${ }^{6}$

El diagnóstico es mediante ultrasonografía, pudiéndose detectar en el tercer trimestre del embarazo. En el RN, cuando el sangrado es significativo, puede evidenciarse clínicamente como una masa palpable abdominal, además de anemia y choque hipovolémico, en los casos más graves. Los hallazgos por ultrasonido son la presencia de una masa ecogénica y compleja, que cuando es grande puede desplazar al riñón. El pronóstico depende de las posibles complicaciones, como calcificaciones anulares o distróficas, o abscesos. ${ }^{7}$

A pesar de ser una entidad que puede ser frecuente, las publicaciones al respecto son limitadas, por lo que decidimos presentar el caso de un RN con HS a fin de refrescar el conocimiento.

\section{PRESENTACIÓN DEL CASO}

RN de sexo masculino; nació en abril del año 2018 en el Hospital Provincial Gineco-Obstétrico "Fe del Valle Ramos" de Manzanillo, Cuba. Fue hijo del primer embrazo de madre de 25 años de edad, profesionista, sin antecedentes de importancia. El embarazo cursó sin complicaciones. Nació a las 39 semanas de gestación por parto; durante el trabajo de parto requirió aplicación de fórceps, presentando depresión moderada, por lo que requirió maniobras de reanimación. El Apgar fue de 1 y 7, por lo que se trasladó a Cuidados Intensivos Neonatales (UCIN). El peso fue de 3,800 gramos, talla de $55 \mathrm{~cm}$ y perímetro cefálico de $37 \mathrm{~cm}$.

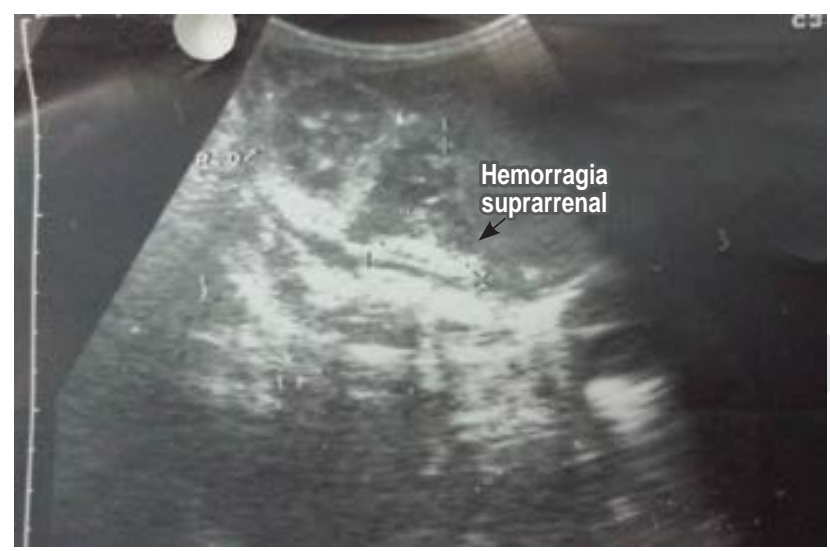

Figura 1: Ultrasonido donde se muestra la hemorragia suprarrenal del lado derecho.

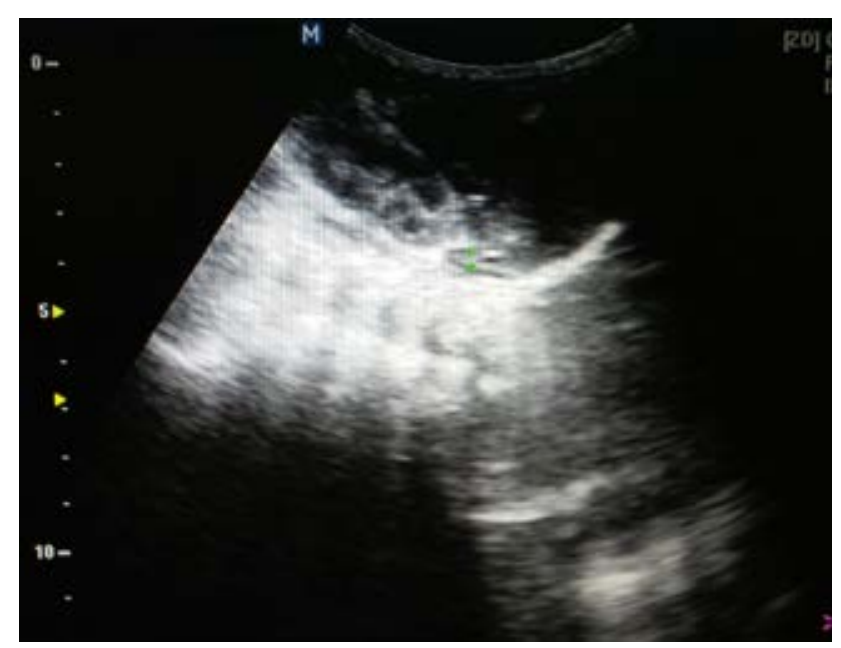

Figura 2: Ultrasonido de glándula suprarrenal derecha sin hemorragia.

Al ingreso a la UCIN se observó con dificultad respiratoria leve, con imitación de movimiento del brazo derecho. Se inicia protocolo de actuación frente a $\mathrm{RN}$ deprimido. Alrededor de las 22 horas de vida se identificó dolor en la exploración física del abdomen, pero sin masa palpable. Por lo que se decide tomar sangre para estudios de laboratorio y realizar ecografía abdominal y transfontanelar.

Se detectó anemia (12.5 gramos de hemoglobina) como único dato anormal. El ultrasonido transfontanelar fue normal. Pero en la ecografía abdominal se observó una imagen compleja a nivel suprarrenal derecha, que medía $44 \times 31 \mathrm{~mm}$, la cual correspondía a HS del lado derecha (Figura 1).

Se decide transfusión de paquete globular, así como transfusión con plasma y aporte de vitamina $\mathrm{K}$. El seguimiento ecográfico de abdomen se realizó cada tres días, observando reabsorción completa de la hemorragia (Figura 2). El RN fue egresado a los 16 días de edad sin complicaciones.

\section{DISCUSIÓN}

La HS constituye una entidad neonatal de relativa frecuencia que, en muchas ocasiones, se desarrolla de forma asintomática, pudiendo pasar desapercibida..$^{4,5}$ La frecuencia de esta entidad es variable; en una serie sobre 3,657 autopsias de $\mathrm{RN}$ se reportó una frecuencia del $0.05 \% .^{8}$

Esta entidad ocurre hasta en $90 \%$ de manera unilateral y, de ellas, el 75-80\% son de lado derecho. En 
cuanto al sexo, en algunas series se describe que hay predominio en varones. ${ }^{6,8,9}$

En general, se manifiesta al nacimiento o durante los primeros días de vida. ${ }^{10}$ Debido a que en el RN la glándula suprarrenal es muy grande, entonces es más vulnerable al daño vascular. ${ }^{11}$ Las manifestaciones clínicas son variables, dependen fundamentalmente del grado de hemorragia y de la cantidad de corteza suprarrenal afectada. ${ }^{2,9}$ Entre las manifestaciones más frecuentes se encuentran anemia, masa abdominal, ictericia y, en menor frecuencia, tumefacción y cambio de color en la piel del escroto. ${ }^{12-14} \mathrm{Si}$ el sangrado es moderado, la sangre permanece en la cápsula suprarrenal. En ocasiones se presenta de forma súbita con choque y distensión entre el primer y tercer día. ${ }^{15}$

\section{REFERENCIAS}

1. Rao $\mathrm{RH}$, Vagnucci $\mathrm{AH}$, Amico JA. Bilateral massive adrenal hemorrhage. Early recognition and treatment. Ann Intern Med. 1989; 110: 227-235.

2. Khuri FJ, Alton DJ, Hardy BE, Cook GT, Churchill BM. Adrenal hemorrhage in neonates: report of 5 cases and review of the literature. J Urol. 1980; 124: 684-687.

3. Fanaroff M. Traumatismos del parto. En: Fanaroff AA, Behrman RE, Martin RJ, Merkatz IR. Enfermedades del feto y del recién nacido. $3^{\underline{a}}$ ed. Buenos Aires: Panamericana; 1985. pp. 295-297.

4. Moshang T, Thornton PS. Trastornos endocrinos del recién nacido. En: Avery GB, Fletcher MA, MacDonald MG, eds.
Neonatología, fisiopatología y manejo del recién nacido. Buenos Aires: Panamericana; 2001. pp. 861-87.

5. Claret I, Jiménez R. Traumatismos fetales y neonatales. En: Cruz M, ed. Tratado de pediatría. Barcelona: Espaxs; 1988. pp. 99-109.

6. Scott EM, Thomas A, McGarrigle HH, Lachelin GC. Serial adrenal ultrasonography in normal neonates. J Ultrasound Med.1990; 9: 279-283.

7. Del Cura RJ, Pedraza S, Gayete A; Sociedad Española de Radiología Médica. Radiología esencial. Buenos Aires: Médica Panamericana. 2010; 119 (II): 1690-1692.

8. Snelling CE, Erb EH. Haemorrhage and subsequent calcification of the suprarrenal. J Pediatr. 1935; 6: 22-41.

9. Bergami G, Malena S, Di Mario M, Fariello G. Echography in the follow-up of neonatal adrenal hemorrhage. The presentation of 14 cases. Radiol Med. 1990; 79(5): 474-478.

10. Navarro OM, Daneman A. Congenital and neonatal conditions. In: Kuhn JP, Caffey J, Haller JO, Slovis TL, Caffey's pediatric diagnostic imaging. 12th ed. Philadelphia: Elsevier Saunders; 2013, pp. 1274-1279.

11. Amoury RA, Barth GW, Hall RT, Rhodes PG, Holder TM, Ashcraft KW. Scrotal ecchymosis: sign of intraperitoneal hemorrhage in the newborn. South Med J. 1982; 75(12): 1471-1475.

12. Duman N, Oren H, Gülcan H, Kumral A, Olguner M, Ozkan H. Scrotal hematoma due to neonatal adrenal hemorrhage. Pediatr Int. 2004; 46(3): 360-362.

13. Palas $\mathrm{J}, \mathrm{AlObaidy} \mathrm{M}$, Ramalho $\mathrm{M}$. Hemiscrotal hematoma in the newborn-don't forget adrenal hemorrhage. J Pediatr. 2015; 166: 491-91.e1.

14. Yarci E, Arayici S, Nur SF, Emre CF, Uras N, Dilmen U. Adrenal hemorrhage presenting as a scrotal hematoma in the newborn. Arch Argent Pediatr. 2015; 113(3): e161-e163/e163.

15. Barrios FA, Barán WM, Sosa AL, Ligia AM, Ávalos S. Manual de atención neonatal. 2 ed. Ministerio de Salud Pública y Bienestar Social. 2016. p. 140. Disponible en: https://www.mspbs.gov.py/dependencias/portal/adjunto/9fac93-MANUALDEATENCINNEONATAL. AUTORIZADOMSPBSRESOLUCINMINISTERIALSGN816.pdf 